\title{
Reflections with Respect to Line and Hyperplane from Quaternionic Point of View
}

\author{
Melek ERDOĞDU ${ }^{1 *}$
}

\begin{abstract}
In this study, the reflections in $\mathbb{E}^{3}$ and $\mathbb{E}^{4}$ are investigated by unit quaternions. Firstly, a linear transformation is defined to describe reflections in $\mathbb{E}^{3}$ with respect to the plane passing through the origin and orthogonal to the quaternion. Then some examples are given to discuss obtained results. Similarly, two linear transformations are stated which correspond to the reflection in $\mathbb{E}^{4}$ with respect to the hyperplane passing through the origin and a reflection with respect to the line in the direction of the quaternion. Finally, the matrix representaions of these reflections are found and the eigenvalues, eigenvectors of them are given to analyse the geometric meaning in terms of the components of the quaternion for each case.
\end{abstract}

Keywords: Quaternions, Reflections, Eigenvalues, Eigenvectors, Rigid Motions.

\section{Kuaterniyon Bakış Açısı ile Doğru ve Hiperdüzlem Boyunca Yansımalar}

ÖZET: Bu çalışmada, $\mathbb{E}^{3}$ ve $\mathbb{E}^{4}$ uzayında yansımalar birim kuaterniyonlar ile incelenmiştir. İlk olarak, $\mathbb{E}^{3}$ uzayında orjinden geçen ve kuaterniyona dik doğrultudaki doğru boyunca yansımayı belirten bir lineer dönüşüm tanımlanmıştır. Ardından, ortaya çıkan sonuçlar örneklendirilmiştir. Benzer şekilde, $\mathbb{E}^{4}$ uzayında orjinden geçen hiperdüzlem ve kuaterniyon doğrultusundaki doğru boyunca yansımalara karşı1ık gelen dönüşümler tanıtılmıştır. Son olarak bu yansıma dönüşümlerinin matris temsilleri elde edilmiş ve her durum için bu özdeğer ve özvekörlerin hesaplanması ile geometrik yorumlar kuaterniyon katsayıları ile analiz edilmiştir.

Anahtar kelimeler: Kuaterniyonlar, Yansımalar, Özdeğerler, Özvektörler, Katı hareketler.

\footnotetext{
${ }^{1}$ Melek ERDOĞDU (Orcid ID: 0000-0001-9610-6229), Necmettin Erbakan University, Deparment of MathematicsComputer Science, Konya, Turkey.

* Sorumlu Yazar/Corresponding Author:Melek ERDOĞDU, e-mail: merdogdu@konya.edu.tr 


\section{INTRODUCTION}

In main problems of physical sciences, the transformations which leave all the distances fixed have an important role. These kind of transformations are called rigid motions and can be descriptive of motion of rigid bodies. It is clear that the identity transformation is a rigid motion and composition of rigid motions is also a rigid motion. Furthermore, any rigid motion has a inverse which is a rigid motion as well. That is, the set of all rigid motions of a space forms a group. Particularly, the group of rigid motions of Euclidean space is designated as Euclidean group. Any rigid motion in Euclidean group is the composite of a linear transformation and a translation. And the linear transformation component of the rigid body is the main discussion which is an orthogonal transformation. An orthogonal transformation $T$ is a linear transformation which has the property $\|T(u)\|=\|u\|$ for all $u$ in the space. Since the orthogonal transformations preserves the length, they preserves the distance as well.

To understand the geometry of rigid motions in Euclidean group, it is obligatory to discuss the structure of orthogonal matrices. A square matrix $T$ of order $n$ with real entries and satisfying the condition, $T^{t} T=T T^{t}=I$, is called an orthogonal matrix in $\mathbb{E}^{n}$ where $I$ is the identity matrix of order $n$. This implies that any matrix $T$ is orthogonal if its inverse is equal to its transpose. Since orthogonal matrices are closed under matrix multiplication and taking inverse, the set of orthogonal matrices forms a group which is called orthogonal group and denoted by $O(n)$. On the other hand, $O(n)$ is a $((n(n-1)) / 2)$ dimensional $C^{\infty}$ manifold on the space of $n \times n$ real matrices, $G L(n, \mathbb{R})$, and has a submanifold tangent space at the identity which means that lie algebra of antisymmetric matrices $o(n)$. Therefore the orthogonal group is a compact lie group (Özdemir and Erdoğdu,
2014; Erdoğdu and Özdemir, 2018; Jadczyk and Szulga, 2016).

The orthogonal group comprises two components. Because the determinant of any orthogonal matrix is either +1 or -1 . The component containing the orthogonal matrices with determinant 1 and also containing the identity matrix is a subgroup of $O(n)$ which is called special orthogonal group and denoted by $S O(n)$. Each elements of $S O(n)$ is called a rotation matrix. The other component containing the orthogonal matrices with determinant -1 is the set of reflections. The main object of this paper is to discuss second kind of orthogonal transformations i.e. reflections with the use of quaternions.

There are several ways to construct orthogonal transformations such as Cayley formula, Rodrigues Rotation's formula, Householder transformations, Clifford algebras and quaternions. Each method works specially for different spaces. For example, some of these methods can be defined for $\mathrm{n}$ dimensional spaces but some of them are useful for only three and four dimensional spaces. But, most interesting way to examine orthogonal transformations is to use Clifford algebras and quaternions (Jadczyk and Szulga, 2014; Zhang, 1997; Friedberg et al., 2003; Roman, 2008).

In the study (Özkaldı and Gündoğan, 2010), the simple reflections, rotations and Cartan Theorem are investigated by using Clifford algebras. Rotations about nonnull axis in Minkowski 3-space are investigated with unit split quaternions in (Özdemir and Ergin, 2006). Then the eigenvalues and eigenvectors of Lorentzian rotation matrices are expressed in terms of corresponding split quaternion and the type of a given Lorentzian rotation (Euclidean or hyperbolic) is determined by the scalar part of the split quaternion in (Özdemir et al., 2014). The Lorentzian rotations, which have null rotation axis, are obtained by using Rodrigues 
and Cayley's formula with pseudo orthonormal frame that include two linearly independent normalized null vectors and a unit spacelike vector and it is proved that every unit timelike split quaternion with null vector part corresponds to a rotation about null axis in (Nesovic, 2016) and rotations on a given null-cone is obtained by using Rodrigues, Cayley and hyperbolic quaternions in (Ünal et al., 2016). At the same time, rotations for a given hyperboloid are investigated in (Şimşek and Özdemir, 2017). Furthermore, elliptical rotations are obtained by Rodrigues Rotation's formula, Cayley formula , union of even number of Hausholder transformations and elliptic quaternions in (Gracia and Thomas, 2017). On the other hand, the rotations in Minkowski space time are studied in (Şimşek and Özdemir, 2016; Erdoğdu and Özdemir, 2015). In a different way, Lorentzian rotations are investigated with Lorentzian matrix multiplications in (Özdemir, 2016; Keçelioğlu et al., 2012). Then, homothetic Cayley formula and transformation are defined Rodrigues and Euler parameters for homothetic motion are obtained in (Gonzalez et al., 2009). Morever, there are also different studies (Şenyurt and Çalışkan, 2018; Şenyurt et al., 2017; Şenyurt and Grill, 2015) on quaternionic curves and surfaces.

This paper consists of three main parts. The first part includes the pricipal properties of quaternions. Then the reflection with respect to plane passing through origin are stated with the use of unit pure quaternions in two different way. Moreover, some examples are given to clarify the discussion. The last part comprises the reflections with respect to the hyperplane passing through the origin and the line in $\mathbb{E}^{4}$ with the use of unit quaternions.

\section{MATERIAL AND METHODS}

In this section, we will give some preliminaries of quaternions.

The set of quaternions is first described by Sir William Rowan Hamilton in 1843 (Wilkins, 1844-1850). Real quaternion algebra

$$
\mathbb{H}=\left\{\mathrm{q}=q_{0}+q_{1} i+q_{2} j+q_{3} k: q_{0}, q_{1}, q_{2}, q_{3} \in \mathbb{R}\right\}
$$

is the foremost member of noncommutative algebras. Here $i, j, k$ are imaginary units which satisfy the product rules in the following table:

\begin{tabular}{|c|c|c|c|c|}
\hline$*$ & 1 & $i$ & $j$ & $k$ \\
\hline 1 & 1 & $i$ & $j$ & $k$ \\
\hline$i$ & $i$ & -1 & $k$ & $-j$ \\
\hline$j$ & $j$ & $-k$ & -1 & $i$ \\
\hline$k$ & $k$ & $j$ & $-i$ & -1 \\
\hline
\end{tabular}

The quaternions can be considered as a generalization of complex numbers (Hacisalihoğlu, 1983). Also, the quaternion algebra is the even subalgebra of the Clifford algebra of the 3-dimensional Euclidean space (Özkaldı and Gündoğan, 2010).

For any quaternion $q=q_{0}+q_{1} i+q_{2} j+q_{3} k$, we may rewrite $q=S(q)+V(q)$ where $S(q)=$ $q_{0}$ and $V(q)=q_{1} i+q_{2} j+q_{3} k$ denote the scalar and vector parts of $q$, respectively. If $S(q)=0$ then $q$ is called pure quaternion. We denote the set of pure quaternions by $\mathbb{H}_{0}$. The conjugate of the 
quaternion $q$ is defined as $\bar{q}=S(q)-V(q)$. The norm of a quaternion $q$ is defined by $\|q\|=$ $\sqrt{q * \bar{q}}=\sqrt{\bar{q} * q}=\sqrt{q_{0}^{2}+q_{1}^{2}+q_{2}^{2}+q_{3}^{2}}$. Therefore, the set of unit quaternions is denoted by

$$
\mathbb{H}_{1}=\left\{q=\left(q_{0}, q_{1}, q_{2}, q_{3}\right): q_{0}, q_{1}, q_{2}, q_{3} \in \mathbb{R}, q_{0}^{2}+q_{1}^{2}+q_{2}^{2}+q_{3}^{2}=1\right\}
$$

Thus, any unit quaternion can be written in the form $q_{0}=\cos \theta+u \sin \theta$ where $u$ is a unit vector satisfying the equality $u * u=-1$ and is called the axis of the quaternion. This representation of a unit quaternion brings out the nost important geometric applications of quaternions, that is any four real numbers are enough to represent a rotation. Only on one condition, the quaternion should be unit (Gracia and Thomas, 2017; Gonzalez et al., 2009; Zhang, 1997).

\section{RESULTS AND DISCUSSION}

Lemma 1 Let $\mathrm{q}$ be a unit pure quaternion. The transformation $\mathrm{T}_{\mathrm{q}}: \mathbb{H}_{0} \rightarrow \mathbb{H}_{0}$ defined by

$$
\mathrm{T}_{\mathrm{q}}(\mathrm{x})=\mathrm{q} * \overline{\mathrm{x}} * \mathrm{q}^{-1}
$$

is a linear transformation which preserving the norm. Here the inverse of unit pure quaternion $\mathrm{q}$ is defined as follows:

$$
\mathrm{q}^{-1}=\frac{\overline{\mathrm{q}}}{\|q\|}=\overline{\mathrm{q}}=-q
$$

Also, $\mathrm{T}_{\mathrm{q}}(\mathrm{x})$ is a pure quaternion satisfying the property $\mathrm{T}_{\mathrm{q}}\left(\mathrm{T}_{\mathrm{q}}(\mathrm{x})\right)=\mathrm{x}$.

Proof. Using properties of the quaternions and $\|\mathrm{q}\|=1$, we see that

$$
\left\|\mathrm{T}_{\mathrm{q}}(\mathrm{x})\right\|=\left\|\mathrm{q} * \overline{\mathrm{x}} * \mathrm{q}^{-1}\right\|=\|\mathrm{q}\|\|\mathrm{x}\|\left\|\mathrm{q}^{-1}\right\|=\|\mathrm{x}\| .
$$

To see that $\mathrm{T}_{\mathrm{q}}(\mathrm{x})$ is linear map, let $\mathrm{a} \in \mathbb{R}$ let $\mathrm{x}$ and $\mathrm{y}$ be any pure quaternions, then,

$$
\begin{aligned}
\mathrm{T}_{\mathrm{q}}(\mathrm{ax}+\mathrm{y}) & =\mathrm{q} *(\overline{\mathrm{ax}+\mathrm{y}}) * \mathrm{q}^{-1}=\mathrm{q} *(\mathrm{ax}+\overline{\mathrm{y}}) * \mathrm{q}^{-1} \\
& =\mathrm{a}\left(\mathrm{q} * \overline{\mathrm{x}} * \mathrm{q}^{-1}\right)+\left(\mathrm{q} * \overline{\mathrm{y}} * \mathrm{q}^{-1}\right)=\mathrm{aT}_{\mathrm{q}}(\mathrm{x})+\mathrm{T}_{\mathrm{q}}(\mathrm{y})
\end{aligned}
$$

Lastly, using the fact that $\mathrm{q}^{2}=(\overline{\mathrm{q}})^{2}=-1$, we find

$$
\begin{aligned}
\mathrm{T}_{\mathrm{q}}\left(\mathrm{T}_{\mathrm{q}}(\mathrm{x})\right) & =\mathrm{T}_{\mathrm{q}}\left(\mathrm{q} * \overline{\mathrm{x}} * \mathrm{q}^{-1}\right)=\mathrm{q} *\left(\overline{\mathrm{q} * \overline{\mathrm{x}} * \mathrm{q}^{-1}}\right) * \mathrm{q}^{-1} \\
& =\mathrm{q} *(\overline{\mathrm{q} * \overline{\mathrm{x}} * \overline{\mathrm{q}}}) * \overline{\mathrm{q}}=(\mathrm{q} * \mathrm{q}) * \mathrm{x} *(\overline{\mathrm{q}} * \overline{\mathrm{q}})=\mathrm{x}
\end{aligned}
$$

Theorem 1 Let $q=q_{1} i+q_{2} j+q_{3} k$ be a unit pure quaternion. The linear transformation $T_{q}: \mathbb{H}_{0} \rightarrow$ $\mathbb{H}_{0}$ represents a reflection with respect to the plane

$$
\mathrm{q}_{1} \mathrm{x}+\mathrm{q}_{2} \mathrm{y}+\mathrm{q}_{3} \mathrm{z}=0
$$


Proof. For any unit pure quaternion $q=q_{1} i+q_{2} j+q_{3} k$, we have

$$
\begin{aligned}
& \mathrm{T}_{\mathrm{q}}(\mathrm{i})=\mathrm{q} *(-\mathrm{i}) *(-\mathrm{q})=\left(-\mathrm{q}_{1}^{2}+\mathrm{q}_{2}^{2}+\mathrm{q}_{3}^{2}\right) \mathrm{i}-2 \mathrm{q}_{1} \mathrm{q}_{2} \mathrm{j}-2 \mathrm{q}_{1} \mathrm{q}_{3} \mathrm{k}, \\
& \mathrm{T}_{\mathrm{q}}(\mathrm{j})=\mathrm{q} *(-\mathrm{j}) *(-\mathrm{q})=-2 \mathrm{q}_{1} \mathrm{q}_{2} \mathrm{i}+\left(\mathrm{q}_{1}^{2}-\mathrm{q}_{2}^{2}+\mathrm{q}_{3}^{2}\right) \mathrm{j}-2 \mathrm{q}_{2} \mathrm{q}_{3} \mathrm{k}, \\
& \mathrm{T}_{\mathrm{q}}(\mathrm{k})=\mathrm{q} *(-\mathrm{k}) *(-\mathrm{q})=-2 \mathrm{q}_{1} \mathrm{q}_{3} \mathrm{i}-2 \mathrm{q}_{2} \mathrm{q}_{3} \mathrm{j}+\left(\mathrm{q}_{1}^{2}+\mathrm{q}_{2}^{2}-\mathrm{q}_{3}^{2}\right) \mathrm{k} .
\end{aligned}
$$

The matrix representation of the linear transformation $\mathrm{T}_{\mathrm{q}}: \mathbb{H}_{0} \rightarrow \mathbb{H}_{0}$ defined by $\mathrm{T}_{\mathrm{q}}(\mathrm{x})=\mathrm{q} * \overline{\mathrm{x}} *$ $\mathrm{q}^{-1}=\mathrm{q} *(-\mathrm{x}) *(-\mathrm{q})=\mathrm{q} * \mathrm{x} * \mathrm{q}$ is obtained as follows:

$$
\mathrm{T}_{\mathrm{q}}=\left[\begin{array}{lll}
-\mathrm{q}_{1}^{2}+\mathrm{q}_{2}^{2}+\mathrm{q}_{3}^{2} & -2 \mathrm{q}_{1} \mathrm{q}_{2} & -2 \mathrm{q}_{1} \mathrm{q}_{3} \\
-2 \mathrm{q}_{1} \mathrm{q}_{2} & \mathrm{q}_{1}^{2}-\mathrm{q}_{2}^{2}+\mathrm{q}_{3}^{2} & -2 \mathrm{q}_{2} \mathrm{q}_{3} \\
-2 \mathrm{q}_{1} \mathrm{q}_{3} & -2 \mathrm{q}_{2} \mathrm{q}_{3} & \mathrm{q}_{1}^{2}+\mathrm{q}_{2}^{2}-\mathrm{q}_{3}^{2}
\end{array}\right]
$$

Then we find $T_{q}$ is orthogonal and $\operatorname{det}\left(\mathrm{T}_{\mathrm{q}}\right)=-\left(\mathrm{q}_{1}^{2}+\mathrm{q}_{2}^{2}+\mathrm{q}_{3}^{2}\right)^{3}=-1$. This means $\mathrm{T}_{\mathrm{q}}$ represents a reflection. The characteristic polynomial of the matrix $\mathrm{T}_{\mathrm{q}}$ can be found as follows:

$$
P_{\lambda}\left(\mathrm{T}_{\mathrm{q}}\right)=\left(\mathrm{q}_{1}^{2}+\mathrm{q}_{2}^{2}+\mathrm{q}_{3}^{2}+\lambda\right)\left(\mathrm{q}_{1}^{2}+\mathrm{q}_{2}^{2}+\mathrm{q}_{3}^{2}-\lambda\right)^{2}
$$

Since $\mathrm{q}_{1}^{2}+\mathrm{q}_{2}^{2}+\mathrm{q}_{3}^{2}=1$, then we obtain eigenvalues of $\mathrm{T}_{\mathrm{q}}$ as $\lambda_{1}=-1$ and $\lambda_{2,3}=1$ with the corresponding eigenvectors

$$
\mathbf{e}_{1}=\left[\begin{array}{l}
q_{1} \\
q_{2} \\
q_{3}
\end{array}\right], \mathbf{e}_{2}=\left[\begin{array}{r}
-q_{2} \\
q_{1} \\
0
\end{array}\right] \text { and } \mathbf{e}_{3}=\left[\begin{array}{r}
-q_{3} \\
0 \\
q_{1}
\end{array}\right]
$$

respectively. Since $\mathrm{T}_{\mathrm{q}}$ represents a reflection with respect to the plane ortogonal to the eigenvector which corresponding to the eigenvalue $\lambda=-1$, we get the proof.

Using a unit pure quaternion $\mathrm{q}$, one can generate a reflection matrix as follows:

$$
\mathrm{T}_{\mathrm{q}}=\left[\begin{array}{lll}
-\mathrm{q}_{1}^{2}+\mathrm{q}_{2}^{2}+\mathrm{q}_{3}^{2} & -2 \mathrm{q}_{1} \mathrm{q}_{2} & -2 \mathrm{q}_{1} \mathrm{q}_{3} \\
-2 \mathrm{q}_{1} \mathrm{q}_{2} & \mathrm{q}_{1}^{2}-\mathrm{q}_{2}^{2}+\mathrm{q}_{3}^{2} & -2 \mathrm{q}_{2} \mathrm{q}_{3} \\
-2 \mathrm{q}_{1} \mathrm{q}_{3} & -2 \mathrm{q}_{2} \mathrm{q}_{3} & \mathrm{q}_{1}^{2}+\mathrm{q}_{2}^{2}-\mathrm{q}_{3}^{2}
\end{array}\right]
$$

On the other hand, we can find a unit pure quaternion corresponding to a given reflection matrix in $\mathbb{E}^{3}$ using the following formulas:

$$
\begin{aligned}
& \mathrm{q}_{1}^{2}=\frac{1}{4}\left(1-\mathrm{T}_{11}+\mathrm{T}_{22}+\mathrm{T}_{33}\right) \\
& \mathrm{q}_{2}=-\frac{1}{2 \mathrm{q}_{1}} \mathrm{~T}_{12} \\
& \mathrm{q}_{3}=\frac{1}{-2 \mathrm{q}_{1}} \mathrm{~T}_{13}
\end{aligned}
$$

for $\mathrm{q}_{1} \neq 0$. When, $\mathrm{q}_{1}=0$, we can find corresponding unit quaternion using the equations: 


$$
\mathrm{q}_{3}=\frac{1}{-2 \mathrm{q}_{2}} \mathrm{~T}_{23} \text { and } \mathrm{q}_{2}^{2}=1-\mathrm{q}_{3}^{2}
$$

Example 1 For the unit pure quaternion $\mathrm{q}=\frac{1}{\sqrt{3}}(0,1,1,1), \mathrm{T}_{\mathrm{q}}$ is

$$
\mathrm{T}_{\mathrm{q}}=\left[\begin{array}{lll}
\frac{1}{3} & -\frac{2}{3} & -\frac{2}{3} \\
-\frac{2}{3} & \frac{1}{3} & -\frac{2}{3} \\
-\frac{2}{3} & -\frac{2}{3} & \frac{1}{3}
\end{array}\right]
$$

This matrix is a reflection matrix in the Euclidean 3 -space, since, $\mathrm{T}_{\mathrm{q}}^{-1}=\mathrm{T}_{\mathrm{q}}$ and $\operatorname{det}_{\mathrm{q}}=-1$.

Example 2 Let's find the reflection with respect to plane the $x+2 y-3 z=0$. The normal vector of the plane $\mathrm{x}+2 \mathrm{y}-3 \mathrm{z}=0$ is $\mathrm{n}=(1,2,-3)$. So, we can take the unit pure quaternion as $\mathrm{q}=$ $\frac{1}{\sqrt{14}}(0,1,2,-3)$. Then, we get

$$
\mathrm{T}_{\mathrm{q}}=\frac{1}{7}\left[\begin{array}{lll}
6 & -2 & 3 \\
-2 & 3 & 6 \\
3 & 6 & -2
\end{array}\right]
$$

Example 3 Consider the reflection matrix

$$
\mathrm{A}=\left[\begin{array}{rrr}
\frac{7}{9} & \frac{4}{9} & \frac{4}{9} \\
\frac{4}{9} & \frac{1}{9} & -\frac{8}{9} \\
\frac{4}{9} & -\frac{8}{9} & \frac{1}{9}
\end{array}\right]
$$

By using equation 2, we get

$$
\mathrm{q}_{1}^{2}=\frac{1}{4}\left(1-\frac{7}{9}+\frac{1}{9}+\frac{1}{9}\right)=\frac{1}{9}
$$

We may take $\mathrm{q}_{1}=\frac{1}{3}$. Then we have

$$
\begin{aligned}
& \mathrm{q}_{2}=-\frac{1}{\frac{2}{3}} \frac{4}{9}=-\frac{2}{3} \\
& \mathrm{q}_{3}=-\frac{1}{\frac{2}{3}} \frac{4}{9}=-\frac{2}{3} .
\end{aligned}
$$

Thus, we obtain $\mathrm{q}=\frac{1}{3} \mathrm{i}-\frac{2}{3} \mathrm{j}-\frac{2}{3} \mathrm{k}$ which means that $\mathrm{A}$ corresponds to a reflection with respect to the plane orthogonal to $\mathrm{n}=\left(\frac{1}{3},-\frac{2}{3},-\frac{2}{3}\right)$. 
Lemma 2 Let $q$ be a unit quaternion. The transformation $\widetilde{\mathrm{T}}_{\mathrm{q}}: \mathbb{H} \rightarrow \mathbb{H}$ defined by

$$
\widetilde{\mathrm{T}}_{\mathrm{q}}(\mathrm{x})=-\mathrm{q} * \overline{\mathrm{x}} * \mathrm{q}
$$

is a linear transformation which preserving the norm. Also, $\widetilde{\mathrm{T}}_{\mathrm{q}}\left(\widetilde{\mathrm{T}}_{\mathrm{q}}(\mathrm{x})\right)=\mathrm{x}$.

Proof. Using properties of the quaternions, we see that the $\widetilde{\mathrm{T}}_{\mathrm{q}}$ preserves the norm.

$$
\left\|\widetilde{\mathrm{T}}_{\mathrm{q}}(\mathrm{x})\right\|=\|-\mathrm{q} * \overline{\mathrm{x}} * \mathrm{q}\|=\|\mathrm{q}\|\|\overline{\mathrm{x}}\|\|\mathrm{q}\|=\|\mathrm{x}\| .
$$

To see that $\widetilde{\mathrm{T}}_{\mathrm{q}}$ is linear, let $\mathrm{a} \in \mathbb{R}$ and let $\mathrm{x}$ and $\mathrm{y}$ be quaternions, then,

$$
\begin{aligned}
& \widetilde{\mathrm{T}}_{\mathrm{q}}(\mathrm{ax}+\mathrm{y})=-\mathrm{q} *(\overline{\mathrm{ax}+\mathrm{y}}) * \mathrm{q} \\
= & -(\mathrm{q} * \overline{\mathrm{ax}} * \mathrm{q})-(\mathrm{q} * \overline{\mathrm{y}} * \mathrm{q}) \\
= & -\mathrm{a}(\mathrm{q} * \overline{\mathrm{x}} * \mathrm{q})-(\mathrm{q} * \overline{\mathrm{y}} * \mathrm{q})=\mathrm{aT}_{\mathrm{q}}(\mathrm{x})+\mathrm{T}_{\mathrm{q}}(\mathrm{y}) .
\end{aligned}
$$

Lastly, using the fact that $\|\mathrm{q}\|^{2}=\overline{\mathrm{q}} * \mathrm{q}=\mathrm{q} * \overline{\mathrm{q}}=1$ and $\overline{\overline{\mathrm{x}}}=\mathrm{x}$ we find

$$
\begin{aligned}
\widetilde{\mathrm{T}}_{\mathrm{q}}\left(\widetilde{\mathrm{T}}_{\mathrm{q}}(\mathrm{x})\right) & =\widetilde{\mathrm{T}}_{\mathrm{q}}(-\mathrm{q} * \overline{\mathrm{x}} * \mathrm{q})=-\mathrm{q} *(\overline{-\mathrm{q} * \overline{\mathrm{x}} * \mathrm{q}}) * \mathrm{q} \\
= & (\mathrm{q} * \overline{\mathrm{q}}) * \mathrm{x} *(\overline{\mathrm{q}} * \mathrm{q})=\mathrm{x} .
\end{aligned}
$$

Theorem 2 Let $q=q_{0}+q_{1} i+q_{2} j+q_{3} k$ be a unit quaternion. The linear transformation $\widetilde{\mathrm{T}}_{\mathrm{q}}: \mathbb{H} \rightarrow$ $\mathbb{H}$ defined by $\widetilde{\mathrm{T}}_{\mathrm{q}}(\mathrm{x})=-\mathrm{q} * \overline{\mathrm{x}} * \mathrm{q}$ represents a reflection with respect to the hyperplane

$$
\mathrm{q}_{0} \mathrm{x}_{1}+\mathrm{q}_{1} \mathrm{x}_{2}+\mathrm{q}_{2} \mathrm{x}_{3}+\mathrm{q}_{3} \mathrm{x}_{4}=0 .
$$

Proof. For any unit quaternion $q=q_{0}+q_{1} i+q_{2} j+q_{3} k$, we have

$$
\begin{gathered}
\widetilde{T}_{q}(1)=-q * 1 * q=-q_{0}^{2}+q_{1}^{2}+q_{2}^{2}+q_{3}^{2}-2 q_{0} q_{1} i-2 q_{0} q_{2} j-2 q_{0} q_{3} k \\
\widetilde{T}_{q}(i)=-q *(-i) * q=-2 q_{0} q_{1}+\left(q_{0}^{2}-q_{1}^{2}+q_{2}^{2}+q_{3}^{2}\right) i-2 q_{1} q_{2} j-2 q_{1} q_{3} k \\
\widetilde{T}_{q}(j)=-q *(-j) * q=-2 q_{0} q_{2}-2 q_{1} q_{2} i+\left(q_{0}^{2}+q_{1}^{2}-q_{2}^{2}+q_{3}^{2}\right) j-2 q_{2} q_{3} k \\
\widetilde{T}_{q}(k)=-q *(-k) * q=-2 q_{0} q_{3}-2 q_{1} q_{3} i-2 q_{2} q_{3} j+\left(q_{0}^{2}+q_{1}^{2}+q_{2}^{2}-q_{3}^{2}\right) k
\end{gathered}
$$

The matrix representation of the linear transformation $\widetilde{\mathrm{T}}_{\mathrm{q}}$ is obtained as follows

$$
:\left[\begin{array}{llll}
-q_{0}^{2}+q_{1}^{2}+q_{2}^{2}+q_{3}^{2} & -2 q_{0} q_{1} & -2 q_{0} q_{2} & -2 q_{0} q_{3} \\
-2 q_{0} q_{1} & q_{0}^{2}-q_{1}^{2}+q_{2}^{2}+q_{3}^{2} & -2 q_{1} q_{2} & -2 q_{1} q_{3} \\
-2 q_{0} q_{2} & -2 q_{1} q_{2} & q_{0}^{2}+q_{1}^{2}-q_{2}^{2}+q_{3}^{2} & -2 q_{2} q_{3} \\
-2 q_{0} q_{3} & -2 q_{1} q_{3} & -2 q_{2} q_{3} & q_{0}^{2}+q_{1}^{2}+q_{2}^{2}-q_{3}^{2}
\end{array}\right]
$$


Then we find $\operatorname{det}\left(\widetilde{\mathrm{T}}_{\mathrm{q}}\right)=-\left(\mathrm{q}_{0}^{2}+\mathrm{q}_{1}^{2}+\mathrm{q}_{2}^{2}+\mathrm{q}_{3}^{2}\right)^{4}=-1$ and $\left(\widetilde{\mathrm{T}}_{\mathrm{q}}\right)^{\mathrm{t}}\left(\widetilde{\mathrm{T}}_{\mathrm{q}}\right)=\mathrm{I}_{4}$. This means $\widetilde{\mathrm{T}}_{\mathrm{q}}$ represents a reflection. Moreover, we obtain eigenvalues of $\widetilde{\mathrm{T}}_{\mathrm{q}}$ as $\lambda_{1}=-1$ and $\lambda_{2,3,4}=1$ with the corresponding eigenvectors

$$
\mathbf{e}_{1}=\left[\begin{array}{l}
q_{0} \\
q_{1} \\
q_{2} \\
q_{3}
\end{array}\right], \mathbf{e}_{2}=\left[\begin{array}{l}
-q_{1} \\
q_{0} \\
0 \\
0
\end{array}\right], \mathbf{e}_{3}=\left[\begin{array}{l}
-q_{2} \\
0 \\
q_{0} \\
0
\end{array}\right], \mathbf{e}_{4}=\left[\begin{array}{l}
-q_{3} \\
0 \\
0 \\
q_{0}
\end{array}\right],
$$

respectively. Since $\widetilde{\mathrm{T}}_{\mathrm{q}}$ represents a reflection with respect to the plane ortogonal to the eigenvector which corresponding to the eigenvalue $\lambda=-1$, we get the proof.

Lemma 3 Let $q$ be a unit quaternion. Then, the transformation $\widetilde{\mathrm{T}}_{\mathrm{q}}: \mathbb{H} \rightarrow \mathbb{H}$ defined by

$$
\widetilde{\mathrm{T}}_{\mathrm{q}}(\mathrm{x})=\mathrm{q} * \overline{\mathrm{x}} * \mathrm{q}
$$

is a linear transformation which preserving the norm. Also, $\widetilde{\mathrm{T}}_{\mathrm{q}}\left(\widetilde{\mathrm{T}}_{\mathrm{q}}(\mathrm{x})\right)=\mathrm{x}$.

Proof. Using properties of the quaternions, we see that the $\widetilde{\mathrm{T}}_{\mathrm{q}}$ ise preserves the norm.

$$
\left\|\widetilde{\mathrm{T}}_{\mathrm{q}}(\mathrm{x})\right\|=\|\mathrm{q} * \overline{\mathrm{x}} * \mathrm{q}\|=\|\mathrm{q}\|\|\overline{\mathrm{x}}\|\|\mathrm{q}\|=\|\mathrm{x}\| .
$$

To see that $\widetilde{\mathrm{T}}_{\mathrm{q}}$ is linear, let $\mathrm{a} \in \mathbb{R}$ and let $\mathrm{x}$ and $\mathrm{y}$ be any quaternions, then,

$$
\begin{aligned}
& \widetilde{\mathrm{T}}_{\mathrm{q}}(\mathrm{ax}+\mathrm{y})=\mathrm{q} *(\overline{\mathrm{ax}+\mathrm{y}}) * \mathrm{q} \\
& =(\mathrm{q} * \overline{\mathrm{ax}} * \mathrm{q})+(\mathrm{q} \overline{\mathrm{y}} \mathrm{q}) \\
& =\mathrm{aT}_{\mathrm{q}}(\mathrm{x})+\mathrm{T}_{\mathrm{q}}(\mathrm{y}) .
\end{aligned}
$$

Lastly, using the fact that $\|\mathrm{q}\|^{2}=\overline{\mathrm{q}} * \mathrm{q}=\mathrm{q} * \overline{\mathrm{q}}=1$ and $\overline{\overline{\mathrm{x}}}=\mathrm{x}$ we find

$$
\begin{aligned}
\widetilde{\mathrm{T}}_{\mathrm{q}}\left(\widetilde{\mathrm{T}}_{\mathrm{q}}(\mathrm{x})\right) & =\mathrm{T}_{\mathrm{q}}(\mathrm{q} * \overline{\mathrm{x}} * \mathrm{q})=\mathrm{q} *(\overline{\mathrm{q} * \overline{\mathrm{x}} * \mathrm{q}}) * \mathrm{q} \\
= & (\mathrm{q} * \overline{\mathrm{q}}) * \mathrm{x} *(\overline{\mathrm{q}} * \mathrm{q})=\mathrm{x} .
\end{aligned}
$$

Theorem 3 Let $q=q_{0}+q_{1} i+q_{2} j+q_{3} k$ be a unit quaternion. The linear transformation $\widetilde{T}_{q}: \mathbb{H} \rightarrow$ $\mathbb{H}$ defined by $\widetilde{\mathrm{T}}_{\mathrm{q}}(\mathrm{x})=\mathrm{q} * \overline{\mathrm{x}} * \mathrm{q}$ represents a reflection with respect to the line in the direction $\left(\mathrm{q}_{0}, \mathrm{q}_{1}, \mathrm{q}_{2}, \mathrm{q}_{3}\right)$.

Proof. For any unit quaternion $\mathrm{q}=\mathrm{q}_{0}+\mathrm{q}_{1} \mathrm{i}+\mathrm{q}_{2} \mathrm{j}+\mathrm{q}_{3} \mathrm{k}$, we have

$$
\begin{gathered}
\widetilde{T}_{q}(1)=q * 1 * q=q_{0}^{2}-q_{1}^{2}-q_{2}^{2}-q_{3}^{2}+2 q_{0} q_{1} i+2 q_{0} q_{2} j+2 q_{0} q_{3} k \\
\widetilde{T}_{q}(i)=q *(-i) * q=2 q_{0} q_{1}+\left(-q_{0}^{2}+q_{1}^{2}-q_{2}^{2}-q_{3}^{2}\right) i+2 q_{1} q_{2} j+2 q_{1} q_{3} k \\
\widetilde{T}_{q}(j)=q *(-j) * q=2 q_{0} q_{2}+2 q_{1} q_{2} i+\left(-q_{0}^{2}-q_{1}^{2}+q_{2}^{2}-q_{3}^{2}\right) j+2 q_{2} q_{3} k
\end{gathered}
$$




$$
\widetilde{T}_{q}(k)=q *(-k) * q=2 q_{0} q_{3}+2 q_{1} q_{3} i+2 q_{2} q_{3} j+\left(-q_{0}^{2}-q_{1}^{2}-q_{2}^{2}+q_{3}^{2}\right) j
$$

The matrix representation of the linear transformation $\widetilde{\mathrm{T}}_{\mathrm{q}}$ is obtained as follows:

$$
\left[\begin{array}{llll}
q_{0}^{2}-q_{1}^{2}-q_{2}^{2}-q_{3}^{2} & 2 q_{0} q_{1} & 2 q_{0} q_{2} & 2 q_{0} q_{3} \\
2 q_{0} q_{1} & -q_{0}^{2}+q_{1}^{2}-q_{2}^{2}-q_{3}^{2} & 2 q_{1} q_{2} & 2 q_{1} q_{3} \\
2 q_{0} q_{2} & 2 q_{1} q_{2} & -q_{0}^{2}-q_{1}^{2}+q_{2}^{2}-q_{3}^{2} & 2 q_{2} q_{3} \\
2 q_{0} q_{3} & 2 q_{1} q_{3} & 2 q_{2} q_{3} & -q_{0}^{2}-q_{1}^{2}-q_{2}^{2}+q_{3}^{2}
\end{array}\right]
$$

Then we find $\operatorname{det}\left(\widetilde{\mathrm{T}}_{\mathrm{q}}\right)=-\left(\mathrm{q}_{0}^{2}+\mathrm{q}_{1}^{2}+\mathrm{q}_{2}^{2}+\mathrm{q}_{3}^{2}\right)^{4}=-1$ and $\left(\widetilde{\mathrm{T}}_{\mathrm{q}}\right)^{\mathrm{t}}\left(\widetilde{\mathrm{T}}_{\mathrm{q}}\right)=\mathrm{I}_{4}$. This means $\widetilde{\mathrm{T}}_{\mathrm{q}}$ represents a reflection. Moreover, we obtain eigenvalues of $\widetilde{\mathrm{T}}_{\mathrm{q}}$ as $\lambda_{1}=1$ and $\lambda_{2,3,4}=-1$ with the corresponding eigenvectors

$$
\mathbf{e}_{1}=\left[\begin{array}{l}
q_{0} \\
q_{1} \\
q_{2} \\
q_{3}
\end{array}\right], \mathbf{e}_{2}=\left[\begin{array}{l}
-q_{1} \\
q_{0} \\
0 \\
0
\end{array}\right], \mathbf{e}_{3}=\left[\begin{array}{l}
-q_{2} \\
0 \\
q_{0} \\
0
\end{array}\right], \mathbf{e}_{4}=\left[\begin{array}{l}
-q_{3} \\
0 \\
0 \\
q_{0}
\end{array}\right]
$$

respectively. Thus $\widetilde{\mathrm{T}}_{\mathrm{q}}$ represents a reflection with respect to the line with the direction $\mathbf{e}_{\mathbf{1}}=$ $\left(\mathrm{q}_{0}, \mathrm{q}_{1}, \mathrm{q}_{2}, \mathrm{q}_{3}\right)$.

\section{CONCLUSION}

In this paper, the reflections in $\mathbb{E}^{3}$ and $\mathbb{E}^{4}$ are studied by unit quaternions. The matrix representations of these reflections are given and the eigenvalues, eigenvectors of them are

\begin{tabular}{|c|c|c|c|}
\hline & Definition & Transformation & Geometric Meaning \\
\hline $\begin{array}{l}q \text { is a unit } \\
\text { pure } \\
\text { quaternion }\end{array}$ & $\mathrm{T}_{\mathrm{q}}: \mathbb{H}_{0} \rightarrow \mathbb{H}_{0}$ & $\begin{aligned} \mathrm{T}_{\mathrm{q}}(\mathrm{x})= & \mathrm{q} * \overline{\mathrm{x}} * \mathrm{q}^{-1} \\
& =\mathrm{q} * \mathrm{x} * \mathrm{q}\end{aligned}$ & $\begin{array}{l}\text { Reflection with respect to the plane } \\
\qquad q_{1} x+q_{2} y+q_{3} z=0\end{array}$ \\
\hline $\begin{array}{l}q \text { is a unit } \\
\text { quaternion }\end{array}$ & $\widetilde{\mathrm{T}}_{\mathrm{q}}: \mathbb{H} \rightarrow \mathbb{H}$ & $\widetilde{\mathrm{T}}_{\mathrm{q}}(\mathrm{x})=-\mathrm{q} * \overline{\mathrm{x}} * \mathrm{q}$ & $\begin{array}{l}\text { Reflection with respect to the } \\
\text { hyperplane } \\
\mathrm{q}_{0} \mathrm{x}_{1}+\mathrm{q}_{1} \mathrm{x}_{2}+\mathrm{q}_{2} \mathrm{x}_{3}+\mathrm{q}_{3} \mathrm{x}_{4}=0\end{array}$ \\
\hline $\begin{array}{l}q \text { is a unit } \\
\text { quaternion }\end{array}$ & $\widetilde{\mathrm{T}}_{\mathrm{q}}: \mathbb{H} \rightarrow \mathbb{H}$ & $\widetilde{\mathrm{T}}_{\mathrm{q}}(\mathrm{x})=\mathrm{q} * \overline{\mathrm{x}} * \mathrm{q}$ & $\begin{array}{l}\text { Reflection with respect to the line in } \\
\text { the direction }\left(q_{0}, q_{1}, q_{2}, q_{3}\right) \text {. }\end{array}$ \\
\hline
\end{tabular}
investigated to analyse the geometric meaning in terms of the components of the quaternion for each case. The following table summarizes the results:

\section{REFERENCES}

Erdoğdu M, Özdemir M, 2015. Cayley Formula in Minkowski Space-time. International Journal of Geometric Methods in Modern Physics, 12.

Erdoğdu M, Özdemir M, 2018. Generating Four Dimensional Rotation Matrices, in progres.
Friedberg AJ, Insel LE, Spence LE, 2003. Linear Algebra. Prentice Hall Pearson Education International, New Jersey.

Gracia AP, Thomas F, 2017. On Cayley's Factorization of 4D Rotations and Applications. Advances in Applied Clifford Algebras, 27: 523-538. 
Gonzalez GA, Aragon JL, Rodriguez-Andrade MA, Verde Star L, 2009. Reflections, Rotations and Pythagorean Numbers. Advances in Applied Clifford Algebras, 19:1-14.

Gürlebeck K, Sprössig W, 1997. Quaternionic and Clifford Calculus for Physicists and Engineers, Series: Mathematical Methods in Practice 1. Wiley.

Hacısalihoğlu H.H, 1983. Hareket Geometrisi ve Kuaterniyonlar Teorisi.Hacisalihoğlu Yayıncılık, Türkiye.

Jadczyk A, Szulga J, 2016. Lorentzian Transformations from Elementary Point of Wiew. The Electronic Journal of Linear Algebra, 31: 794-813.

Jadczyk A, Szulga J, 2014. A Comment on "On the Rotation Matrix in Minkowski Space-time" by Özdemir and Erdoğdu. Reports on Mathematical Physics, 74: 39-44.

Keçelioğlu O, Özkaldı S, Gündoğan H, 2012. Rotations and Screw Motion with Timelike Vector in 3-Dimensional Lorentzian Space. Advances in Applied Clifford Algebras, 22:1081-1091.

Nesovic E, 2016. On Rotation About Lightlike Axis in Three Dimensional Minkowski Space. Advances in Applied Clifford Algebras, 26: 237-251.

Özdemir M, 2016. An Altenative Approach to Eliptical Motion. Advances in Applied Clifford Algebras, 26: 279-304.

Özdemir M, Ergin AA, 2006. Rotations with unit timelike quaternions in Minkowski 3-space. Journal of Geometry and Physics, 56: 322-336.

Özdemir M, Erdoğdu M, Şimşek H, 2014. On the Eigenvalues and Eigenvectors of a Lorentzian Rotation Matrix by Using Split Quaternions. Advances in Applied Clifford Algebras, 24: 179-192.

Özdemir M, Erdoğdu M, 2014. On the Rotation Matrix in Minkowski Space-time. Reports on Mathematical Physics, 74: 27-38.
Özkald1 S, Gündoğan H, 2010. Cayley Formula, Euler Paremeters and Rotations in 3Dimensional Lorentzian Space. Advances in Applied Clifford Algebras, 20: 367-377.

Roman S, 2008. Advanced Linear Algebra, Graduade text in mathematics. Springer, USA.

Şenyurt S, Çalışkan A, 2018. The Quaternionic Expression of Ruled Surfaces. Filomat, 32: 5753-5766.

Şenyurt S, Cevahir C, Altun Y, 2017. On Spatial Quaternionic Involute Curve A New View. Advances in Applied Clifford Algebras, 27: 1815-1824.

Şenyurt S, Grill L, 2015. Spherical Indicatrix Curves of Spatial Quaternionic Curvesç Applied Mathematical Sciences, 9: 4469-4477.

Şimşek H, Özdemir M, 2017. Rotations on Lightcone in Minkowski 3-Space. Advances in Applied Clifford Algebras, 27: 2841-2853.

Şimşek H, Özdemir M, 2016. Generating Hyperbolical Rotation Matrix for a Given Hyperbolid. Linear Algebra and Its Applications, 496: 221-245.

Ünal D, Güngör MA, Tosun M, 2016. Homethetic Cayley Formula and Its Applications. Advances in Applied Clifford Algebras, 26: 809-824.

Wilkins DR, 1844-1850. On Quaternions or On A New System of Imaginaries in Algebra by William Rowan Hamilton. Philosophical Magazine.

Zhang F, 1997. Quaternions and Matrices of Quaternions. Linear Algebra and Its Applications, 251: 21-57. 